\title{
Students individual engagement in GIS
}

Madsen, Lene Møller; Christiansen, Frederik V; Rump, Camilla Østerberg

Published in:

Journal of Geography in Higher Education

DOI:

10.1080/03098265.2014.910758

Publication date:

2014

Document version

Early version, also known as pre-print

Citation for published version (APA):

Madsen, L. M., Christiansen, F. V., \& Rump, C. Ø. (2014). Students individual engagement in GIS. Journal of Geography in Higher Education, 38(2), 251-265. [Selected for publication as Chapter 14: Students' individual engagement in GIS. In Haigh, M., Cotton, D. and Hall, T. (eds.) (2016): Pedagogic Research in Geography Higher Education, Routledge.]. https://doi.org/10.1080/03098265.2014.910758 
This article was downloaded by: [Copenhagen University Library]

On: 29 April 2014, At: 07:56

Publisher: Routledge

Informa Ltd Registered in England and Wales Registered Number: 1072954 Registered

office: Mortimer House, 37-41 Mortimer Street, London W1T 3J H, UK

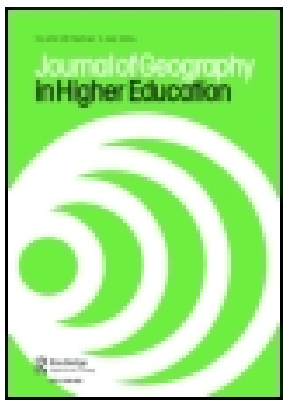

\section{J ournal of Geography in Higher Education}

Publication details, including instructions for authors and subscription information:

http:// www.tandfonline.com/loi/ cjgh20

\section{Students individual engagement in GIS}

Lene Møller Madsen ${ }^{a}$, Frederik Christiansen ${ }^{a} \&$ Camilla Rump ${ }^{a}$

a Department of Science Education, University of Copenhagen, Østre Voldgade 3, Copenhagen K DK-1350, Denmark

Published online: 24 Apr 2014.

To cite this article: Lene Møller Madsen, Frederik Christiansen \& Camilla Rump (2014): Students individual engagement in GIS, J ournal of Geography in Higher Education, DOI: 10.1080/03098265.2014.910758

To link to this article: http:// dx. doi.org/ 10.1080/ 03098265.2014.910758

\section{PLEASE SCROLL DOWN FOR ARTICLE}

Taylor \& Francis makes every effort to ensure the accuracy of all the information (the "Content") contained in the publications on our platform. However, Taylor \& Francis, our agents, and our licensors make no representations or warranties whatsoever as to the accuracy, completeness, or suitability for any purpose of the Content. Any opinions and views expressed in this publication are the opinions and views of the authors, and are not the views of or endorsed by Taylor \& Francis. The accuracy of the Content should not be relied upon and should be independently verified with primary sources of information. Taylor and Francis shall not be liable for any losses, actions, claims, proceedings, demands, costs, expenses, damages, and other liabilities whatsoever or howsoever caused arising directly or indirectly in connection with, in relation to or arising out of the use of the Content.

This article may be used for research, teaching, and private study purposes. Any substantial or systematic reproduction, redistribution, reselling, loan, sub-licensing, systematic supply, or distribution in any form to anyone is expressly forbidden. Terms \& Conditions of access and use can be found at http://www.tandfonline.com/page/termsand-conditions 


\title{
Students individual engagement in GIS
}

\author{
Lene Møller Madsen*, Frederik Christiansen and Camilla Rump \\ Department of Science Education, University of Copenhagen, Østre Voldgade 3, Copenhagen K \\ DK-1350, Denmark
}

(Received 16 March 2013; final version received 3 November 2013)

\begin{abstract}
This paper develops two sets of concepts to theorize why students engage differently in Geographical Information Systems (GIS). These theoretical concepts are used as an analytical lens to explore empirical data on the experiences and engagement of students enrolled in an undergraduate GIS course in planning and management. The analysis shows that both the theoretical perspectives and the custom and didactical contract are important to understand students' engagement in GIS. However, it is the personal desiderata that are the key to understanding the students' different engagement. Further, a temporal dimension and contextual awareness are important in understanding students' engagement in a broader perspective.
\end{abstract}

Keywords: geographical information systems; Kuhn; didactical contract

\section{Introduction}

I don't get the overview ... I don't feel like: GIS can do this and that ... I am not using it in the things I'm doing ... You need to really push yourself to use it ... it is easy not to use it (Emilie).

I think it's extremely satisfying ... that is something that really bothers me about the university studies that you are not allowed to use your creativity. So when you suddenly get the opportunity [with GIS] ... to me that's just fantastic (Maria).

The above quotes are from two students both enrolled in a university course on geographical information systems (GIS) in planning and management. They are examples of how students often engage very differently with GIS. We shall address and unfold these and other students' experiences of the specific course in GIS, as well as establish an analytical framework for understanding more generally students' different engagement in GIS. Engagement is a contested concept within educational research. It ranges from a dichotomous view that students are either engaged or not to a multidimensional view of engagement that combines diverse aspects of students' experiences. In between are notions of engagement as a measured quantity, a categorical view where engagement comprises a series of unordered categories, and a naive view where the concept of engagement is not given further scrutiny than as a well-accepted and well-understood concept (Solomonides, Reid, \& Petocz, 2012). In this paper we apply a multidimensional view of engagement that: "describe various relationships between the student, study and the institution, including the campus" (Solomonides et al., 2012, p. 16) acknowledging that how engagement is created or sustained is extremely complex, and many different factors influence the development of engagement also in relation to the students perspectives of a future work.

*Corresponding author. Email: 1mmadsen@ind.ku.dk 
In the research of GIS in higher education, we have within the last 30 years and with good reasons been occupied with finding and exploring new and innovative ways of promoting and teaching GIS. Learning with GIS can provide students with spatial thinking skills, giving them a tool for geographical analysis and enabling them to be critical citizens. Quite a number of papers on these issues have been published with different foci on, for example, teaching methods, learning outcomes, and interacting with communities (e.g., Barcus \& Muehlenhaus, 2010; Demirci, 2011; Elwood, 2009; Lee \& Bednarz, 2009; Madsen \& Nielsen, 2013; Rød, Larsen, \& Nilsen, 2010; Unwin, Foote, Tate, \& DiBiase, 2011).

This paper is not about how to teach a course in GIS, exploring new innovative fields of teaching or concerned with the learning outcome. Instead it wonders about why it can be that students seem to experience courses in GIS so very differently as the two above quotes show. It does so with the purpose of getting a deeper understanding of students' engagement in GIS during their geography higher education. Why do some students grasp the opportunities provided by GIS, while others decide to avoid future encounters with GIS? It is based on a genuine puzzlement of why students entering a specific undergraduate GIS course at the Department of Geoscience and Natural Resource Management at the University of Copenhagen engage so differently. The GIS course in planning and management is, in terms of pedagogy, well designed. It is a hands-on, problem-based course with the central problem revolving around an authentic planning problem. It allows students to work with an open problem allowing for multiple solutions, in contrast to the often pre-structured step-by-step laboratory exercises used in many GIS courses (Madsen \& Nielsen, 2013; West, 2012). Therefore, the course appears exemplary of characteristic uses of GIS in the Danish labor market (Madsen, Christiansen, \& Rump, 2009), and in pedagogical organization of student activities aiming at the expected student outcomes. Despite the careful design, students engage very differently in the course.

To address this perplexity we turn to Kuhn and his description of an essential tension between convergent and divergent thinking in the practice of science (Kuhn, 1959). In his few papers on science education, we find arguments for transforming his ideas on the phenomenal world of scientists to the phenomenal world of students. However, in order to address our puzzlement more specifically to the organization of the learning process, we also draw on Balacheff (1999) and his distinction between custom and didactical contract. We develop two sets of theoretical concepts (theoretical perspective and shared and personal desiderata; custom and didactical contract) which are unfolded in the next sections. These concepts are then used in the empirical part of the paper as an analytical lens to unfold the experiences and engagement of students enrolled in the GIS course in planning and management. In this way we aim to address our research question: how can we theorize why students engage so differently in a specific GIS course?

\section{Theoretical concepts to understand students' engagement}

\section{Theoretical perspective and shared and personal desiderata}

In this section we introduce the first set of theoretical concepts for our analysis based on ideas from Thomas Kuhn's philosophy of science. Using Kuhn's theory may at first seem an odd choice, as Kuhn's main concern was with scientific development and our concern is with student development. We find, however, that Kuhn's discussion of values (Kuhn, 1977) and cognitive model for theory choice (Kuhn, 1959, 1974) have a relatively clear analog in students' choices to pursue or not to pursue GIS. We will return to this analogy below. Further, we find that Kuhn's theory provides a fruitful starting point for studying 
students' engagement because it provides a unique combination of sociological, cognitive, and phenomenological insights into a relatively coherent theoretical position.

The first theoretical concept that we want to describe is what we have termed theoretical perspective. By "theoretical perspective" we refer to paradigmatic ways of conceiving problems and phenomena within the field. In a number of his writings, Kuhn developed a philosophical and cognitive theory that could explain the apparent paradox of how educations that were by and large convergent in nature (Kuhn, 1959) could still lead to tradition-shattering periods of divergence (Kuhn, 1970, 1974, 1977). Kuhn's idea was that the problem-solving activities that take up most of science education provide the students with an arsenal of exemplars, on the basis of which he or she may proceed to new (but similar) problems. However, this "reasoning by exemplars" is not a rule-governed activity, but based on fundamental perceptions of similarity or dissimilarity with previously encountered problems. The absence of rules mean that even though students learn to solve problems and classify phenomena (i.e., make judgments that $\mathrm{Y}$ is an instance of X) in fairly much the same way, they do not do so because of a mutual agreement on the necessary and sufficient conditions for X membership. This means that in "normal" situations, the scientist works within the framework of the paradigm, extending his/her knowledge from exemplars to new phenomena and domains. Mostly, scientists will arrive at the same judgments, even though the processes leading to the judgments need not be the same for different individuals. This process of "reasoning from exemplars" we will term "theoretical perspective".

However, using the theoretical perspective is not enough to arrive at judgments in all cases. In situations where scientists are to choose between two rivaling theories, they cannot make use of their normal scientific methods and techniques. The paradigmatic "way of seeing" phenomena that the scientist has been socialized into does simply not suffice to determine the answer to which theory to choose. In such cases the scientist has to rely on more general values (Kuhn, 1977). Kuhn identifies five such general values: accuracy in accounting for phenomena, consistency (both internally), and with respect to other generally accepted theories, broadness of scope, simplicity, and fruitfulness. Kuhn regards these values as relatively stable over time, and a part of the cultural heritage of the natural sciences as such. Thus, these values are shared by all and have later been termed desiderata (Hempel, 1988; the term was later adopted by Kuhn).

The desiderata are quite imprecise individually. For instance, two individuals who both subscribe to the same value of accuracy may still come to disagree about whether one theory is more accurate than another. In addition, there is no fixed way in which to determine how the different desiderata should be weighed with respect to each other in specific evaluations of theory (Kuhn, 1977). The scientist's personal experiences, preferences, and values will determine how the scientist will end up weighing the desiderata. Thus, there are actually two sets of values at play here. One set is what we will term shared desiderata, and designates values which are shared by members of the scientific community (e.g., the list of values above). The other set of values are determined by the scientist's personal background and affinities, and we shall term these personal desiderata (see Hoyningen-Huene, 1993, pp. 150-151).

Thus, overall we distinguish between two different ways of arriving at judgments. The first has to do with what is determined by the paradigm and results in judgments that are typically unanimous within the scientific community. This is what we have termed the theoretical perspective. The other is based on desiderata that can be either shared within the community or personal. Desiderata based judgments will generally differ from individual to individual in the community. 
Let us now use Kuhn's description of scientists practice to explore our aim of understanding students' engagement in GIS. We will of course necessarily have to reinterpret what we mean by the terms theoretical perspective and shared and personal desiderata now that we are considering students rather than scientists. In the student setting, the theoretical perspective involves the types of judgments that are facilitated or made possible on the basis of the knowledge acquired through "reasoning by exemplars" through their education. That is, having followed a course in GIS, a student will be able to make specific types of analyses based on the types of analyses encountered previously, and (ideally at least) extend this knowledge to new situations. By shared desiderata we refer to values and norms which are shared in the specific student body. For instance, some student environments are very competitive, while others more oriented toward student collaboration. In some environments student cherish qualitative research, in others quantitative. In some environments, education is perceived by most students as a service provided to them, in others as something that the students essentially contribute to, etc. Finally, the personal desiderata have to do with the values pertaining to the individual rather than the student body. Examples could be choosing to work with a particular region because you lived there as a child or because you want to move there, choosing elective courses because you want to strengthen your knowledge within subjects in which you have a personal interest, etc. In the following we shall designate the theoretical perspective $T$, shared desiderata $S$, and personal desiderata $P$.

We turn now to how these three different perspectives may have different roles to play at different times based on the context. Table 1 gives a schematic overview of the influence of the concepts (theoretical perspective, shared and personal desiderata) during a specific course for an individual student. A student in the situation of choosing an elective course (for example, in GIS) relies mainly upon shared $S$ and personal desiderata $P$, whereas prior theoretical knowledge is bracketed or suppressed [T], because it is of little help in the concrete situation. When working in a course, the theoretical perspective becomes important and is added to or developed. This is indicated by the apostrophe $T\left(T^{\prime}\right)$. At this time the personal desiderata become bracketed due to the convergent nature of science education. There is little room for personal judgments, they have to be suppressed. However, even though $P$ is suppressed in the course of work, the result after the course is nonetheless a development both in personal desiderata $P^{\prime}$ and in the theoretical knowledge $\left[T^{\prime}\right]$. The meaning of $S$ and $S^{\prime}$ refers to custom, and custom plus didactical contract, respectively. Further explanation is given in the following sections.

In many situations during their studies, students make choices about how they are going to specialize, one of them being when they are choosing elective courses. As with the scientist facing a choice of theory, the student relies in such situations mainly on shared and personal desiderata and only to a limited degree on the theoretical perspective in these situations of choice. The theoretical perspective simply does not provide answers to the question "which course to choose", and cannot provide valuable justifications.

Table 1. Schematic view of the development of theoretical perspectives, shared and personal desiderata for a prototypical student in a GIS course.

\begin{tabular}{lccc}
\hline & Theoretical perspective & Shared desiderata & Personal desiderata \\
\hline Electing a course & {$[T]$} & $S$ & $P$ \\
Working in a course & $T^{\prime}$ & $S^{\prime}$ & {$[P]$} \\
After a course & {$\left[T^{\prime}\right]$} & $S$ & $P^{\prime}$ \\
\hline
\end{tabular}


Therefore, in situations of electing a course, we may say that the theoretical perspective is suppressed ([T] in Table 1). As the student begins working in a course, however, the theoretical perspective becomes crucial, whereas the personal desiderata are bracketed ( $T$ and $[P]$ in Table 1 ) because the student will have to accept and subscribe to the teaching and learning activities in the course, and more or less leave behind his or her personal preferences and interests. The level of suppression of $P$ depends on the students' ability to relate their personal preferences and interest to the teaching and learning activities, e.g., free choices of topics. However, due to the convergent nature of science suppression will occur. After the course, both the theoretical perspective and the personal desiderata will have been changed ([T $]$ and $P^{\prime}$ in Table 1). Because of the limited scope of the theoretical perspective, we have again suppressed it as before the course. The distinction between $S$ and $S^{\prime}$ needs further elaboration in terms of another set of concepts which can extend the understanding of shared desiderata; namely custom and didactical contract.

\section{Custom and didactical contract}

The second set of theoretical concepts that we address is that of custom and didactical contract. Above we distinguished between shared desiderata and personal desiderata. Based on Kuhn's description of the shared desiderata as relatively stable over time, we here denote shared desiderata as the students' shared and general values, norms, beliefs, practices, and behavior in relation to their education. These are in play and used when a student chooses a course ( $S$ in Table 1). In accordance with Kuhn's description of shared desiderata, Balacheff (1999) uses the term custom as: "a set of obligatory practices... established as such by their use and which, in the majority of cases, is established implicitly" (Balacheff, 1999, p. 25). He further argues that customs are not confined to a particular class but extend to particular levels of schooling (for example, customs related to 7th grade geography teaching). Thus, Balacheff's concept of custom and our concept of shared desiderata are interchangeable. In addition, Balacheff (1999) makes a distinction between custom and didactical contract, which we can use to understand what happens when a student starts working in a course. In such situations the student subscribes to both the custom/shared desiderata and to a set of explicit but local and temporary rules governing the teaching activities in the specific course. These rules relate mainly to the teaching and learning activities and assessment of the course (e.g., deliveries, work formats, and examination) and are termed the didactical contract. This contract does not overrule the general and tacit shared desiderata $S$, but adds to them $\left(S^{\prime}\right.$ in Table 1$)$. The didactical contract is explicit, has a local character, and is negotiated for a particular task that requires the rules for the social functioning of the class to be defined locally and in a new way. Thus, the students come to the class with certain shared desiderata, but must then subscribe to a specific didactical contract involving acceptance of specific types of teaching and learning activities defined by the teacher (though sometimes in negotiation with the students). However, given the temporary nature of the didactical contract, the student will rely on the custom only after the course.

Consequently, in our analogy to Kuhn's description of scientists and scientific development, we may define three evaluative moments that come into play in different ways at different times as an essential tension in students development; namely the theoretical perspective $T$, the shared desiderata $S$ including the distinction between custom and didactical contract, and the personal desiderata $P$. In the next sections, we shall outline how we can use these concepts to understand students' engagement in relation to a specific GIS course. 


\section{The empirical setting of the study}

\section{The learning environment}

At the Department of Geoscience and Natural Resource Management at the University of Copenhagen, teaching in GIS has been part of the curriculum for human and physical geographers since the late 1980s. The development of different GIS courses as well as the present teaching practice has been addressed in Madsen and Rump (2012) and Madsen and Winsløw (2009).

The GIS course in planning and management is taken by around 30 students each year and is an optional course within the M.Sc. program in Geography and Geoinformatics. In Denmark there is no tradition of employing students with only a bachelor degree. Hence very few students leave the university with a bachelor degree; most graduate with a Master's degree. The GIS course is part of the elective courses within the first 3 years of the 5-year M.Sc. program which means that the geography students attending the course may not yet have chosen whether they are to become either human or physical geographers. However, the course can also be taken by students from other studies (for example, biology or natural resource management) as either a bachelor or a master course and further by people already having a master as a paid course (open university course). It is a 7-week course taking up half of the students' study activities in the period. The course introduces central GIS tools for working with planning and management issues such as network analysis, digital records, and web-based communication systems (ArcGIS, version 9.1 was used). The course curriculum is organized around the students' group based problem-solving activities. The students in the course work on three consecutive assignments in which central functions of GIS are explored in relation to semi-authentic problems. The last assignment is somewhat larger than the other two, and constitutes the basis for the oral examination. The topic of this assignment is a question of where to place a new institution (for example, kindergarten, school, and shopping mall), given the distribution of existing institutions and other relevant variables such as municipal objectives from district plans and population density in the municipality. The analysis should be based on data-sets provided to the students, and different types of GIS analyses should be made. The students' proposal should be outlined in a WebGIS for location of the institution, so decision-makers and/or citizens could get an impression of the different aspects of the problem by adding or subtracting different layers, for instance buffer zones based on noise data, or relevant age distributions.

\section{Participants and interviews}

In a specific year between 2002 and 2012 (in order to secure anonymity of the participants, the exact year is not given), we followed eight students participating in the GIS course in planning and management. Observations in the classroom were made twice in the beginning of the course and contacts with the students were established. The eight students were selected with the purpose of securing a diverse group of students in order to optimize the opportunity to learn the most from the case as discussed in Stake (2005). The group of interviewed students consisted of five men and three women. Six were geography students of which three were either taking quite a number of courses or a whole master in other disciplines (political sciences, economics, and development studies). One student was from biology and one was from geography at one of the other universities in the Copenhagen area. 
We performed individual qualitative interviews with each student on two occasions. The first round was made before the students' commenced working on the final assignment, and the second interview shortly after the oral examination in the course. The interviews were conducted with an open interview guide. In the first interview, students were asked to describe why they had chosen the course, their conception of the problem that they were going to work with, and were asked to consider its relevance for their future use of GIS. In the second interview, the students were asked to explain how they had solved the problem in the final assignment and what their general conceptions of the course, the problem, and the examination were. Further, they were asked to elaborate on their motivation for working with GIS, and how they saw GIS as part of their future professional life. These questions were used because we wanted them to inform our two sets of theoretical concepts (shared and personal desiderata and custom and didactical contract) to understand students' engagement as described in the following analytical approach. Each interview took around $1 \mathrm{~h}$. All eight students passed the course with grades ranging from A to $\mathrm{C}$ on the European Credit Transfer and Accumulation System scale, that is, all students performed well in the course.

All 16 interviews were made in Danish, taped, and subsequently transcribed with acceptance of the interviewees. We have chosen verbatim translations rather than linguistically correct ones. All interviews were performed by the first two authors together; one of us being an insider to the subject matter of GIS and geography as a discipline and one of us being an outsider (Adriansen \& Madsen, 2009).

\section{The analytical approach}

The objective of the empirical part of this paper is to investigate the interplay between students' expression of the influence of their theoretical perspective $T$, shared desiderata $S$ (including the distinction between custom and didactical contract), and personal desiderata $P$ while taking the GIS course, and how the interactions between these three elements affected the students' engagement in using GIS for geographical problem solving.

Having interviews early on in the course made it possible for us to understand the students' personal desiderata $P$, their preliminary conceptions of $T$ in relation to GIS, their understanding of custom $S$, the didactical contract $S^{\prime}$, and their engagement in relation to using GIS. Further, having interviews after the examination with the same students enabled us to analyze their understanding of the relationship between their experience with the teaching and learning activities $S^{\prime}$ and resulting content learning $T^{\prime}$, how their personal desiderata had been changed as a result of the course (from $[P]$ to $P^{\prime}$ ), and how their engagement in relation to GIS had possibly changed during the course. Based on our multidimensional view on engagement, we have for each interview estimated the subject's engagement in relation to using GIS in their future work, irrespective of whether it is related to their study or a future job situation. Here we have been inspired by self-efficacy beliefs and outcome expectancy beliefs in relation to using GIS, i.e., the student's beliefs that he or she will be able to accomplish specific tasks involving GIS, and the conception of being in an environment that is conducive to using these capabilities (Bandura, 1982). In estimating the level of engagement, we have aimed at not having a precise measure of their engagement, but in making a distinction between low, medium, or high engagement based on a holistic (in the sense of taking as many factors into considerations as possible) assessment of each interview. The engagement scores in each interview transcription have been assigned independently by the authors and discussed until a consensus has been reached. 
Table 2. Eight students' way through the undergraduate GIS course in planning and management described in terms of engagement and relationships between $S, P$, and $T$ (see text for description of letters).

\begin{tabular}{|c|c|c|c|}
\hline Student & $\begin{array}{l}\text { Engagement } \\
\text { during course }\end{array}$ & $\begin{array}{l}\text { Engagement } \\
\text { after course }\end{array}$ & $\begin{array}{c}\text { Relationships } \\
\text { between } S, P \text {, and } T\end{array}$ \\
\hline \multicolumn{4}{|c|}{ Stories of $T^{\prime}$ influencing $P^{\prime}$} \\
\hline Søren & High & High & $T^{\prime}+[P]$ \\
\hline Thomas & High & High & $T+[P]$ \\
\hline Emilie & Medium & Low & $T-[P]$ \\
\hline Anette & High & Medium & $T-[P]$ \\
\hline Carl & High & Medium & $T-[P]$ \\
\hline \multicolumn{4}{|c|}{ Story of external factor $E$ and $T^{\prime}$ influencing $P^{\prime}$} \\
\hline Charlotte & Low & High & $T+E+[P]$ \\
\hline \multicolumn{4}{|c|}{ Stories of $S^{\prime}$ influencing $P^{\prime}$} \\
\hline Maria & Medium & High & $S^{\prime}+[P]$ \\
\hline Ida & Medium & High & $S^{\prime}+[P]$ \\
\hline
\end{tabular}

\section{Findings}

Table 2 shows the results of our analysis of the interviews with students. It shows the eight students' way through the course described in terms of engagement and relationships between $S, P$, and $T$. The symbols + and - signify a positive or a negative reinforcement between two elements. Thus, $S^{\prime}+[P]$ means positive reinforcement between didactical contract and suppressed personal preferences. The $E$ for Charlotte denotes external factors of relevance.

\section{The relevance of geographical content: five stories of $\mathbf{T}^{\prime}$ influencing $\mathbf{P}^{\prime}$}

Although they have quite different backgrounds, Søren and Thomas share many views on GIS and the role they see GIS playing in their future. Both are just finishing their studies, and both see a GIS-related job in a municipality (administrative unit in Denmark) as an attractive job opportunity. Both find the course and the assignments in the course highly relevant for them and in general. Søren has a genuine interest in the geographical planning issues involved and has chosen to look at the placement of a new school. He is excited about the possibilities for modeling the future that the GIS program provides. He also thinks that the problem resembles closely the types of problems you would get if working in a municipality, and finds this motivating. Thomas has chosen to look at the placement of a shopping mall instead. His justification for this is based on his reading of actual local development plans he found at the municipalities' website on his own initiative. He also finds the authenticity of the problem important: "I think it is a funny ... lets-pretend-it-is... It works much better than just following some tutorial. Yes I like that".

Thus, for both Søren and Thomas, there was a very good match between their personal desiderata $P$ and the theoretical perspective adopted in the course $T$. Both liked the way the teaching content was organized into a semi-authentic planning problem that students had to work on $S^{\prime}$, because it matched well with their job aspirations $P$.

In contrast to Thomas and Søren who are just finishing their studies, Emilie is in her third year of study. Emilie also had specific goals with the course based on her personal desiderata $P$. She wanted to be better at using GIS, so she could use it in her bachelor project about coast lines in the Wadden Sea. However, while she does find the authentic 
problem a relevant planning problem, Emilie feels that working with this problem does not help her to use GIS in her Bachelor project or elsewhere: "I had thought that it would be broader... it is all very focused on this specific problem about Tårnby municipality, and it's all right, but it is just a small niche of what GIS can do". In the second interview, Emilie seems more reluctant toward using GIS because of its technicality and says that she would need urging and guidance in order to use GIS in the future. Thus for Emilie the reinforcement between personal desiderata $P$ and the theoretical perspective used in the course $T$ was negative.

Anette wants to finish her bachelor in geography. She has strong political interests, and she has just applied for a job in one of the unions. Her interest in GIS is that it would be a good tool to know whether she wants to work with planning in the future. However, during the GIS course she has realized that she wants to work with the political level and not the practical level at which the course operates. She finds it unsatisfying that there is so little focus on the planning and analysis behind the problem, and so much focus on the visual aspects of GIS. She finds the authentic problem about Tårnby municipality too simplistic. After the examination she tells us that she did not get the job in the union and when she looks back at the GIS course she thinks that it is nice that she now knows something about the potential uses of GIS, but she does not expect to make use of them herself in her further studies or after graduation, as she expects to operate at the political level rather than the technical. So also for Anette, the match between $P$ and $T$ turns out to be negative.

The negative relationship between $P$ and $T$ is also the case for Carl, another bachelor student. He enters the GIS course with no specific ideas about this particular GIS course, but with a strong interest in GIS in general. He basically wants to take every GIS-related course available, and he has some ideas about creating GIS-based software applications in the future. Some years ago he studied computer science but got fed up by the abstract work and changed to geography in order to work with some more for him real problems. He sees GIS as a possibility to combine his interest in computers with authentic problem solving. However, he finds the planning problem in the GIS course quite boring. However, because of his interest in GIS he uses the course to explore the functionalities of the software. His final assignment displays this - on the one hand he has used functionalities in GIS not used by any of the other participants, but on the other hand there is a relatively glaring mistake in his assignment. At the examination he got a lower grade, and in the final interview he is having doubts about whether GIS can be used to bridge his interest between computer and real problems.

For Carl, Anette, and Emilie, the theoretical perspective $T$ did not appeal to them, and they did not feel that the course helped them pursue their interests in GIS. The course did not help them develop their engagement in relation to GIS, and it has changed their personal desiderata in distancing them from using GIS. Emilie could see the idea in working with the semi-authentic problem, but felt it to be somewhat irrelevant. Anette felt that it was too simplistic and did not reflect the complexity of political decision-making. Carl had real difficulties in seeing the idea in the assignment at all.

\section{How external factors have relevance: a story of how external factors $\mathbf{E}$ and $\mathbf{T}^{\prime}$ positively reinforce $\mathbf{P}^{\prime}$}

Charlotte has taken the GIS course because she has heard it was an easy course to pass. She is a geography student and sees GIS as a tool: "it is good to have" but she does not find it intrinsically engaging. She does not link GIS to geographical problems. She talks enthusiastically about her job where she works with a program named GeoGIS. She has 
been typing in data into a database, but does not see that it has any relationship with GIS. She finds the authentic problem in the course okay (authentic because it is a planning problem that a municipality is likely to deal with), but does not see it as a real problem for her (since for her to be "real" it must be personally relevant). In the second interview, she has just been defending her bachelor project with good results and is very happy. She has decided to use some more time on her job and she has had some conversations about her future work tasks. In this process she has realized that there is a GIS function attached to the database she has been working with, and that GeoGIS is in fact a GIS. She is now fascinated with how GIS can be used to solve geographical problems (in this case soil pollution). Charlotte has during the time of the course engaged in GIS due to a combination of the course and her job functions. Thus, working with the course content $T$ has made her see and pursue new opportunities in her job situation, and in turn changed her personal perspective on GIS. Thus, Charlotte's story is similar to Søren's and Thomas's stories, with the important difference being that the theoretical perspective $T^{\prime}$ has been strengthened by factors outside the course while the course is running.

\section{The relevance of geographical methods: two stories of $\mathbf{S}^{\prime}$ influencing $\mathbf{P}^{\prime}$}

Ida and Maria both liked the GIS course a lot and both are really engaged in GIS. Maria is actually studying biology, but has previously taken other GIS courses. In general she has found it difficult to keep her motivation for studying, but the GIS courses have been something of a revelation to her. She wants to take all the GIS courses available and she has no specific expectations for this course. She finds GIS very appealing: "the fact that you can work creatively and get a product and all the time follow the process". It is a great challenge to work with and she finds it great fun. Her assignment is quite a work of art. Taking GIS courses has meant that Maria has found the joy in attending class again. In relation to the authentic problem, she does not find the planning problem in itself very appealing but the tools and the intentions with the course she finds highly relevant, it is the way of learning in the GIS courses that appeals to her (the relationship between $P$ and $S^{\prime}$ ).

Ida's story is similar. Ida is a geography student in the third year and she has strong interests in economy. She says about the way of learning: "I think it is fun to analyze and see ... we can sit with it for hours ... clicking the layers on and off and putting photos underneath ... and I could easily do it just for fun". In the second interview, Ida describes why she would like a job where GIS plays a role:

I would like that ... I think it is really fun to work with, so if I could sit with this much of the day, that would be lovely ... I also love to sit and work in Excel and make graphs and such things ... If there is something where I can use something with some numbers, I'd like that.

While the first quote is quite geographically oriented, the second quote highlights the fundamental joy Ida has in engaging in the type of work with a computer and "something where I can use something with numbers".

For Ida and Maria, then, it is not so much the planning aspect and the specific problem that they like or can relate to (as for Søren and Thomas), but rather the way that the learning is organized and the mode of learning in the course, that is $S^{\prime}$. They find it motivating and rewarding to transform numbers into something useful and accessible, and GIS is one instrument (among others) that allows them to do that. In that sense there is a very positive reinforcement between $S^{\prime}$ and $P$ for these two students. 


\section{Discussion}

In the following we discuss how the analysis using the introduced theoretical concepts can address our puzzlement of why students engage so differently in a specific GIS course.

First, our analysis and results show that it is mainly the shared and personal desiderata that determine in which way the students in this study are going to engage with GIS. Second, this being so, the personal desiderata are actually suppressed most of the time in the actual GIS course despite the students possibility for choosing a topic in the final assignment. Hence, in order to understand why the students engage so differently with GIS, it becomes important to consider the longitudinal interplay between theoretical perspectives, shared desiderata, personal desiderata, and possible other factors both before, during, and after a student's interaction with a specific GIS learning experience. This is unfolded in the next sections.

\section{Theoretical perspective and proximity of goals}

In terms of fulfilling the learning objectives or acquiring the intended theoretical knowledge of the GIS, all of the interviewed students solved the authentic planning problem adequately and all did well on the examination (despite the fact that one got a lower grade than the others). However, it was very different how the problem was conceived among the students, that is, how the theoretical perspective of the course influenced the students' personal desiderata. Of particular interest in this respect are the "negative" stories of Carl, Emilie, and Anette. As discussed in the introduction, the course is in many ways pedagogically well designed and authentic with respect to likely job situations. However, as we have seen, none of the three students experienced the problem as authentic or relevant for their immediate goals or their engagement in GIS. Each of them had personal and relevant ideas about potential uses of GIS for geographical problem solving, but the course did not help them pursue these goals. For these students the authentic problem tended to extinguish their interest in GIS rather than igniting it. They were not capable of relating the authentic problem to their personal desiderata, and while they all did well in the course, the course was not conducive for their engagement with GIS in the future. The curricular implications of these observations could be that it would have been more relevant to do a course which helped these students pursue their ideas about GIS use in relation to their upcoming bachelor projects.

For Charlotte, Søren, and Thomas, it was also the relationship between the theoretical perspective of the course and their personal desiderata which was crucial for their engagement in GIS. However, for them it was an igniting experience. Both Søren and Thomas were almost finishing their studies and experienced the authentic planning problem as highly relevant and were fueling their interest in GIS and their job aspirations. For Charlotte it was her job outside her study that secured a positive relationship between the theoretical perspective and her personal desiderata. For all three students it was factors outside the control of the GIS course that created their engagement.

\section{Learning methods: customs and contracts}

For Maria and Ida, what they liked about the course was not the theoretical perspective of the course and thereby the authentic planning problem, but rather their engagement in learning methods adopted in the course. They were engaged in GIS due to the relationship between their personal desiderata and the custom and didactical contract in the course. Maria feels that her potentials are realized far better in the way the learning activities are 
organized in the GIS course than in other courses. As she put it: "my motivation for coming to the teaching was quite different than for the teaching at the Biology Department". Ida describes how she has been taking an unusual number of courses in the political science and economy programs, for a geography student. She engages in GIS by connecting it to her interest in economy, in practice by relating her work with GIS with her work with Excel, which she much enjoys. For these two students it seems that no matter what the theoretical perspective had been in the course, they would have been engaged.

\section{Implications for future teaching in GIS}

The above analysis shows that during the GIS course, the students are suppressing their personal desiderata $P$ and need to subjugate to the theoretical perspective and the shared desiderata as unfolded in the learning methods of the course. Our analysis of eight students shows that those who get engaged in GIS are those who are able to relate their initial personal desiderata with either the theoretical perspective adopted in the course or the learning methods adopted in the course. For the students who are able to relate to the theoretical perspective in the course, it is factors outside the course that are significant; either a forthcoming job or an existing study-related job. For the students who are engaged through the learning methods in the course, it is the relationship with a known learning method or an attractive unknown learning method that is significant for creating the relationship. The students who are not engaged in GIS during the course however stay on and take the examination and even do well on it. To elaborate on this is beyond the scope of this paper; however, it could be a question of deferred gratification as described in Johannsen (2012) or that the students are able to bridge the gap between their expectations to the GIS course and their experiences with the course through different negotiation strategies (Holmegaard, Madsen, \& Ulriksen 2013).

The practical implications of this for teaching and learning GIS are:

(1) We need to keep teaching GIS in a pedagogically well-designed way with handson and authentic problem solving because it engages certain students. However, we need to address the fact that the engagement is linked to the students' personal values, meaning that elements outside the learning situation define and modify the engagement. Therefore, in order to sustain engagement, the proximity of students' goals must be closely tightened with the theoretical perspective. In our empirical study it would imply that we should either:

(a) Consider turning the GIS course into a graduate course instead of it being an undergraduate course. In this way proximity to the goal of getting a job would be closer (because bachelor degrees are not generally seen as sufficient in the Danish labor market - in countries where bachelor degrees are a way to the labor market, this would not apply).

(b) keep it as an undergraduate course and open up for students to work with their own ideas in relation to the content in the course.

(2) We need to allow for and fuel the creativity in learning methods used in GIS courses because it creates connections between theoretical perspectives and some students' personal values igniting an engagement in GIS.

(3) The temporal aspect of the suppressing of personal desiderata and theoretical perspectives before, during, and after the course presented in our analysis has implications for teaching as well. Most of the feedback to students about their individual learning trajectories takes place during the actual teaching. However, if 
we want to influence students' long-term engagement, the time to do that might not be during courses but "in between" courses. There are many ways such guidance can be accomplished - making students keep a learning portfolio describing aims, ambitions, and engagement, and provide feedback on that, or providing student mentoring where teachers counsel students about their aspirations and how to best meet them. Such moments for meta-reflections by students might benefit immensely by keeping them "at a distance" from the didactical contracts of specific courses.

(4) But something can also be done within the course framework. Prior to the start of the course, students can be asked (e.g., as an e-learning assignment) to describe their interest in GIS, why have they chosen the course, and what are their thoughts about using GIS in the future? This will inform the teachers about the thoughts and aspirations of the students with respect to GIS, and individual needs and engagements may be met as the course progresses. Such meta-reflections could also be incorporated into the evaluation of specific courses after the examination.

\section{Conclusion}

We began this paper based on our puzzlement of why students seem to experience courses in GIS so very differently and with the purpose of getting a deeper understanding of students' engagement in GIS during their geography higher education. If we as geography faculty members agree that GIS can give students valuable competencies and see GIS as an integral part of becoming a geographer, then it seems very reasonable questions to pursue.

The analysis of 16 interviews with eight students in a GIS course shows that both the theoretical perspective (in our case capabilities obtained with respect to solving an authentic planning problem) and the learning methods in the GIS course were significant for the students' engagement in relation to GIS. However, they were not acting at the same time for individual students and they did not fulfill all students' interest in GIS. What instead was significant was the theoretical perspective and learning methods in relation to what we have called the students personal desiderata, meaning that in order for students to see problems and learning methods as authentic, they must also be seen as relevant to the student's individual and immediate and future goals and ambitions.

As for the theoretical part of this paper, we found that by developing and using the theoretical concepts of theoretical perspective, shared and personal desiderata in an empirical analysis of a specific GIS course, we became aware that studying students' engagement in relation to GIS needs a longitudinal component. At different times in relation to the students' tasks of electing a course, working in a course, and having finished a course, the perspectives have different emphasis. Thus, a temporal dimension and contextual awareness of understanding students' engagement in GIS are important.

There can be little doubt that all of the introduced concepts can be elaborated and explored further. However, our analysis of student learning in relation to the GIS course has shown that the Kuhnian analogy and the rudimentary distinction between the different types of student evaluative perspectives provide us with a useful tool for analyzing student learning trajectories and the efficacy of curriculum design.

\section{Acknowledgments}

This study is based on observations and interviews with students at the Department of Geoscience and Natural Resource Management, University of Copenhagen. We are indebted to all of them for having shared their time and thoughts with us not only in relation to the GIS course but also in 
relation to their personal feelings and perceptions concerning their present and future study and life trajectories. Also we want to thank the teacher for letting us observe the teaching practice, the students' engagement in the course, and discuss our queries into the course. Finally we want to thank four anonymous reviewers for their valuable comments that significantly improved the paper. All names used in the article are fictitious and all usual disclaimers apply.

\section{References}

Adriansen, H. K., \& Madsen, L. M. (2009). Implications of doing insider interviews: Studying geography and geographers. Norwegian Journal of Geography, 93, 145-153.

Balacheff, N. (1999). Custom and contract: Two registers of didactical interactions. The Mathematics Educator, 9, 23-29.

Bandura, A. (1982). Self-efficacy mechanism in human agency. American Psychologist, 37, $122-147$.

Barcus, H., \& Muehlenhaus, B. (2010). Bridging the academic - public divide in GIS and cartography: A framework for integrating community partnerships in the classroom. Journal of Geography in Higher Education, 34, 363-378.

Demirci, A. (2011). Using geographic information systems (GIS) at schools without a computer laboratory. Journal of Geography, 110, 49-59.

Elwood, S. (2009). Integrating participatory action research and GIS education: Negotiating methodologies, politics and technologies. Journal of Geography in Higher Education, 33, $51-65$.

Hoyningen-Huene, P. (1993). Reconstructing scientific revolutions - Thomas S. Kuhn's philosophy of science. Chicago, IL: University of Chicago Press.

Hempel, C. G. (1988). On the cognitive status and the rationale of scientific methodology. Poetics Today, 9, 5-27.

Holmegaard, H. T., Madsen, L. M., \& Ulriksen, L. (2013). A journey of negotiation and belonging: Understanding students' transitions to higher education science and engineering. Cultural Studies in Higher Education. doi:10.1007/s11422-013-9542-3

Johannsen, B. F. (2012). Attrition and retention in university physics. A longitudinal qualitative study of the interaction between first year students and the study of physics (PhD-thesis). Department of Science Education, University of Copenhagen 2012.

Kuhn, T. S. (1959). The essential tension. In CW Taylor (Ed.), The third (1959) University of Utah research conference on the identification of scientific talent (pp. 162-174). Salt Lake City, UK: University of Chicago Press.

Kuhn, T. S. (1970). Structure of scientific revolution (2nd ed.). Chicago, IL: University of Chicago Press.

Kuhn, T. S. (1974). Second thoughts on paradigms. In F. Suppe (Ed.), The Structure and scientific theories (pp. 459-482). Urbana: University of Illinois Press.

Kuhn, T. S. (1977). Objectivity, value judgment, and theory choice. In T. S. Kuhn (Ed.), The essential tension (pp. 320-339). Chicago, IL: University of Chicago Press.

Lee, J., \& Bednarz, R. (2009). Effect of GIS learning on spatial thinking. Journal of Geography in Higher Education, 33, 183-198.

Madsen, L. M., Christiansen, F. V., \& Rump, C. Ø (2009, March 22-24). Development of personal desiderata while learning GIS. In A. Bisel \& M. U. Garib (Eds.), Frontiers in Science Education Research: Proceedings of the Frontiers in Science Education Research Conference (pp. 441450). Famagusta, North Cyprus: Eastern Mediterranean University Press.

Madsen, L. M., \& Nielsen, T. T. (2013). Learning to do geography? University students posing questions in GIS laboratory exercises. Norwegian Journal of Geography, 67, 157-161.

Madsen, L. M., \& Rump, C. (2012). Considerations of how to study learning processes when students use GIS as an instrument for developing spatial thinking skills. Journal of Geography in Higher Education, 36, 97-116.

Madsen, L. M., \& Winsløw, C. (2009). Relations between teaching and research in physical geography and mathematics at research-intensive universities. International Journal of Science and mathematics Education, 7, 741-763.

Rød, J. K., Larsen, W., \& Nilsen, E. (2010). Learning geography with GIS: Integrating GIS into upper secondary school geography curricula. Norwegian Journal of Geography, 64, $21-35$. 
Solomonides, I., Reid, A., \& Petocz, P. (2012). The nature of the elephant: Metaphors for student engagement. In I. Solomonides, A. Reid, \& P. Petocz (Eds.), Engaging with learning in higher education (pp. 475-485). Faringdon, UK: Macquarie University Research Online.

Stake, R. E. (2005). Qualitative case studies. In N. K. Denzin \& Y. S. Lincoln (Eds.), The sage handbook of qualitative research (3rd ed.) (pp. 443-466). Thousand Oaks, CA: Sage.

Unwin, D. J., Foote, K. E., Tate, N. J., \& DiBiase, D. (Eds.). (2011). Teaching geographic information science and technology in higher education. Chichester: John Wiley \& Sons.

West, E. (2012). Where to begin? Getting started teaching GIS\&T. In D. J. Unwin, K. E. Foote, N. J. Tate, \& D. DiBiase (Eds.), Teaching geographic information science and technology in higher education (pp. 145-157). Chichester: John Wiley \& Sons. 